\section{La voie Nrf2 \\ en pathologie \\ respiratoire}

Anne Boutten, Delphine Goven, ćlise Artaud-Macari, Marcel Bonay

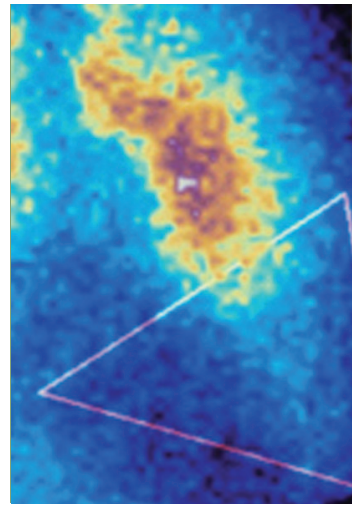

D. Goven, $\varepsilon$. Artaud-Macari : Inserm U700; Université Paris 7, Faculté de médecine Denis Diderot-site Bichat, BP416, 16, rue Henri Huchard, 75870 Paris, France. A. Boutten : Inserm U700; Université Paris 7 ,

Faculté de médecine Denis Diderot-site Bichat, Paris ; service de biochimie, Hôpital Bichat, 16, rue Henri Huchard, 75870 Paris, France.

M. Bonay : Inserm U700 ; Service de physiologie-explorations fonctionnelles, Hôpital

sa forme réduite et les vitamines $A$, $\varepsilon$ et $C$; (2) enzymatiques : la superoxyde dismutase (SOD) et les enzymes de détoxification des xénobiotiques appelées enzymes de conjugaison de phase II (EPII, intervenant après les enzymes d'hydroxylation de phase I du système cytochrome $\mathrm{P} 450$ par exemple) Ambroise Paré - Assistance Publique Hôpitaux de Paris, 9, avenue Charles de Gaulle, 92104 Boulogne Cedex France et Université de Versailles SaintQuentin-en-yvelines, France. marcel.bonay@apr.aphp.fr anne.boutten@bch.aphp.fr par Nrf2 et l'intérêt potentiel d'une thérapeutique ciblant ce facteur. <

En contact direct avec l'environnement extérieur, les voies respiratoires et le poumon sont la première cible des oxydants contenus dans les polluants atmosphériques, notamment la fumée de cigarette [1]. Les cellules du système respiratoire peuvent également être exposées à des oxydants générés localement par les cellules intervenant dans la réponse inflammatoire, en particulier les macrophages et les polynucléaires neutrophiles. Ces cellules sont capables de produire en excès des espèces réactives de l'oxygène (ROS pour reactive oxygen species) et de l'azote (RNS pour reactive nitrogen species) potentiellement délétères pour le poumon à cause du stress oxydant et des dommages tissulaires qu'elles engendrent lors de la phagocytose des particules inhalées. Pour faire face à ces agressions oxydantes, le poumon possède de nombreuses défenses qui font intervenir des mécanismes variés: (1) non enzymatiques comme le glutathion dans
(Tableau 1). La régulation des gènes

codant pour les EPII est contrôlée notamment par l'intermédiaire d'une séquence régulatrice appelée élément de réponse antioxydant (ARE pour antioxidant responsive element) présente dans la région 5' de leur site promoteur. L'activation de l'ARE nécessite la translocation nucléaire et la liaison d'un facteur de transcription. En fonction du type cellulaire et du stimulus, différents types de facteurs de transcription : nuclear factor erythroid-2-related factor 2 (Nrf2), activator protein-1 (AP-1) ou nuclear factor- $\mathrm{B} B(\mathrm{NF}-\mathrm{KB})$ par exemple peuvent aboutir à l'activation de l'ARE. Bien que délétères à fortes concentrations, les ROS et RNS participent à l'homéostasie cellulaire à concentration physiologique. En effet, ROS et RNS sont largement impliquées dans les réponses cellulaires physiologiques telles que les défenses anti-infectieuses, les voies de signalisation intracellulaires et la réponse mitogène. Les ROS sont des éléments modulateurs des états d'activation et de différenciation cellulaires [2, 3] par l'intermédiaire des kinases/phosphatases et de leurs seconds messagers. Les ROS sont également capables de promouvoir la croissance cellulaire voire de favoriser un phénotype cancéreux, ou, à l'inverse, d'induire la sénescence et l'apoptose [4-7]. Contrairement aux ROS, l'implication des EPII et de leur voie de régulation via Nrf2 dans l'état d'activation cellulaire est peu connue [4, 5]. Leur modulation permettrait d'envisager de nouvelles thérapeutiques. 


\begin{tabular}{|c|c|c|}
\hline Mécanisme d'action & Gènes Nrf2 dépendants & Fonction \\
\hline \multirow[t]{2}{*}{ Homéostasie du glutathion (GSH) } & - Glutathion-S-transférase (GST)* & - Conjugue le GSH aux agents chimiques \\
\hline & - Glutathion peroxydase (Gpx) & $\begin{array}{l}\text { - Réduit le peroxyde d'hydrogène et alkyle les } \\
\text { hydroperoxydes }\end{array}$ \\
\hline \multirow[t]{2}{*}{ Métabolisme des xénobiotiques } & - NADPH quinone oxydoréductase $1(\mathrm{NQO}-\mathrm{l}) \star$ & $\begin{array}{l}\text { - Réduit les quinones et antioxydants endogènes, } \\
\text { détruit l'anion superoxyde }\end{array}$ \\
\hline & - Époxyde hydrolase $1(\varepsilon P H X-1) \star$ & - Catalyse l'hydrolyse des époxydes \\
\hline \multirow[t]{2}{*}{ Réponse au stress/métabolisme du fer } & - Hème oxygénase $1(\mathrm{HO}-1) \star$ & $\begin{array}{l}\text { - Catabolise l'hème en monoxyde de carbone, } \\
\text { biliverdine et fer libre }\end{array}$ \\
\hline & - Ferritine & - Séquestre le fer libre \\
\hline
\end{tabular}

Tableau I. Enzymes de phase II, antioxydants et cytoprotecteurs faisant intervenir Nrf2. * : enzymes de phase II.

\section{La voie de signalisation du facteur Nrf2}

Le stress oxydant induit des voies de signalisation sensibles au statut redox (mitogen-activated protein kinase [MAP kinase] et la cascade du nuclear factor $K B[N F K B]$ ) qui activent l'expression de cytokines pro-inflammatoires, de chimiokines et de récepteurs de molécules d'adhésion. Le stress oxydant induit également des réponses protectrices par l'intermédiaire de l'ARE. L'activation de l'ARE nécessite la translocation dans le noyau de facteurs de transcription tels que les NFE2-related factors (Nrfl-2 et 3). Nrfl et Nrf2 sont inducteurs de gènes antioxydants alors que Nrf3 serait inhibiteur. De plus, Nrfl jouerait un rôle critique lors du développement [8]. Les kinases ERK (extracellular signal-regulated kinase), JNK (c-Jun N-terminal kinases) et p38 sont impliquées dans la phosphorylation de Nrf2 qui module non seulement sa localisation nucléaire et son interaction avec les séquences régulatrices en stabilisant Nrf2 [9], mais également son externalisation du noyau, mettant fin à l'activation des séquences modulatrices. Nrf2 entre en compétition avec un autre facteur de transcription, Bachl (BTB et CNC homology 1, basic leucine zipper transcription factor 1 ) retenu dans le cytoplasme par l'hème. Après formation d'un hétérodimère avec les petites protéines Maf, Jun, Fos ou ATF (activating transcription factor), Nrf2 interagit avec l'ARE pour induire l'expression d'EPII telles que : Ia NADPH quinone oxydoréductase-1 (N $00-1)$, l'époxyde hydrolase-1 (EPHX-1), l'hème oxygénase-1 (HO-1), les glutathion-S-transférases (GST), etc. Ces enzymes ont des effets cytoprotecteurs, antioxydants et détoxifiants. Les EPII contribuent à la synthèse et au recyclage des thiols et facilitent l'élimination des métabolites secondaires de détoxication des xénobiotiques et en particulier des agents carcinogènes vis-à-vis desquels $\mathrm{N} \rho \mathrm{O}-\mathrm{l}$ et les GST ont un rôle protecteur [10]. De plus, des protéines de stress comme HO- 1 et les chaînes $H$ et $L$ de la ferritine sont protectrices visà-vis des agressions oxydantes [11]. H0-l dégrade le noyau hème, potentiellement oxydant, et génère des composés antioxydants dont le monoxyde de carbone (CO), la bilirubine et le fer libre. Ce dernier induit la synthèse de ferritine à l'origine de sa séquestration, le substituant à des réactions radicalaires ultérieures [11] (Tableau I). En l'absence d'oxydant, le facteur Nrf2 reste séquestré dans le cytoplasme fixé au cytosquelette par la protéine Kelch-like erythroid cell-derived protein CNC homologyassociated protein 1 (Keapl) [9], et il y est dégradé rapidement par le protéasome (Figure 1).

\section{Nrf2 en pathologie}

La réponse au stress utilisant la voie de signalisation Nrf2/Keapl-Bachl est impliquée dans la protection contre le vieillissement, la neurodégénérescence, la carcinogenèse, l'hépatotoxicité, les maladies cardiovasculaires et les maladies respiratoires comme la bronchopneumopathie chronique obstructive (BPCO) et l'emphysème, l'asthme, la fibrose et les agressions pulmonaires aiguës [12]. En pathologie respiratoire, des études récentes ont confirmé chez l'homme les résultats obtenus dans les modèles animaux.

\section{Modèles animaux}

Les souris déficitaires en $\mathrm{Nrf2}\left(\mathrm{Nrf2}^{-/}\right)$se développent normalement mais présentent une susceptibilité accrue à divers types d'agressions aiguës ou chroniques [9]. Au niveau pulmonaire, ces modèles ont permis d'explorer certains déterminants de la susceptibilité à la fibrose, l'emphysème, l'asthme et au syndrome de détresse respiratoire aiguë (Tableau II). Les souris $\mathrm{Nrf2}^{-/-}$sont également utiles pour vérifier la spécificité des molécules visant à restaurer Nrf2, actuellement proposé comme cible thérapeutique en pathologie respiratoire [5].

\section{Nrf2 en pathologie respiratoire humaine}

Nrf2 est exprimé chez l'homme de manière ubiquitaire dans le poumon, mais principalement par les cellules 


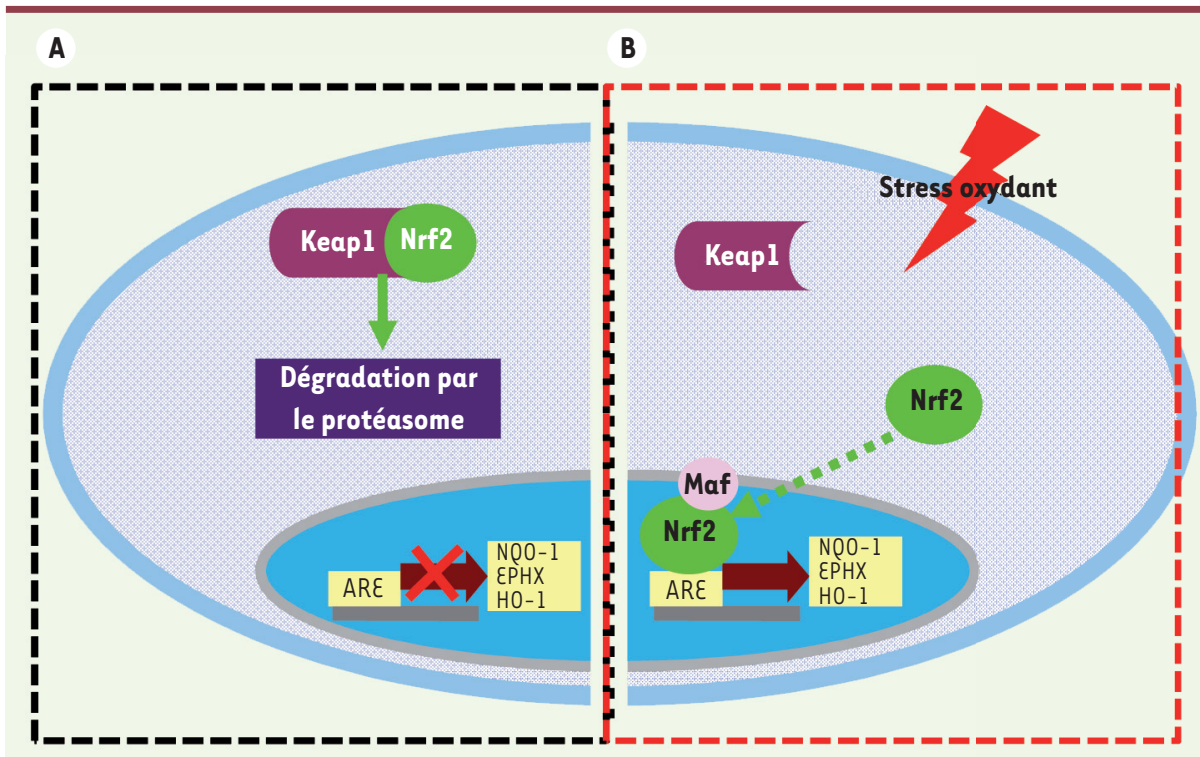

Figure 1. Voie de signalisation de nuclear factor erythroid-2-related factor 2 (Nrf2) et transcription des gènes codant pour les enzymes de phase II. A. En conditions basales, la protéine kelch-like $\mathrm{ECH}$-associated protein 1 (Keapl) lie Nrf2 qui est ainsi dégradé par le protéasome. La translocation de Nrf2 dans le noyau est faible. B. En réponse au stress oxydant, Nrf2 est libéré de Keapl et migre dans le noyau où il s’hétérodimérise avec d'autres facteurs de transcription comme les protéines Maf pour induire la transcription des gènes dépendants de l'élément de réponse antioxydant (ARE), ce qui rétablit ainsi l'équilibre oxydants/antioxydants. NQO-1 : NADPH quinone oxydoréductase-1; EPHX-1 : époxyde hydrolase-1; HO- 1 : hème-oxygénase-1.

\begin{tabular}{ll}
\hline Stimulus & $\boldsymbol{\varepsilon}$ ffets \\
Bléomycine & $\nearrow$ Inflammation et fibrose pulmonaire \\
Hyperoxie & $\nearrow$ Lésions épithéliales alvéolaires, \\
& $\nearrow$ Inflammation macrophagique \\
& $\nearrow$ Perméabilité vasculaire \\
\hline Fumée de cigarette & $\nearrow$ Emphysème pulmonaire : début plus précoce et lésions \\
& $\nearrow$ emphysémateuses plus étendues \\
Élastase & $\nearrow$ Inflammation bronchoalvéolaire \\
\hline Ovalbumine & $\nearrow$ Réponse asthmation et emphysème pulmonaire \\
\hline Endotoxines & $\nearrow$ Inflammation pulmonaire, odème et choc septique \\
\hline
\end{tabular}

Tableau II. Susceptibilité pulmonaire des souris $\mathrm{Nrf}^{-/-}$aux agressions aiguës et chroniques.

épithéliales et les macrophages alvéolaires. Nrf2 joue un rôle essentiel de protection contre le stress oxydant induit par les polluants environnementaux et les toxiques comme la fumée de tabac. La voie Nrf2/ Keapl-Bachl est impliquée dans la réparation pulmonaire normale et pathologique qui s'accompagne d'une production importante de ROS.

\section{$B P C O$ et emphysème pulmonaire}

L'intoxication tabagique est un facteur de risque important de développement de l'emphysème pulmonaire, problème majeur de santé publique responsable en grande partie de la morbidité et de la morta- lité de la BPCO. Les macrophages alvéolaires, dont le nombre est augmenté dans le poumon des fumeurs [13], acquièrent, en réponse à l'exposition à la fumée de cigarette, des phénotypes polarisés Ml ou M2 associés respectivement à une inflammation ou un remodelage tissulaire. Une reprogrammation en faveur d'un phénotype M2 contribuerait à la pathogénie de la BPCO [14]. Les macrophages alvéolaires contribuent pour une large part au déséquilibre de la balance protéase-antiprotéase, au remodelage tissulaire et à l'inflammation pulmonaire [15] dans l'emphysème en produisant notamment des ROS impliqués dans le déséquilibre de la balance oxydant-antioxydant. Goven et al. ont montré pour la première fois dans une étude clinique de patients atteints d'emphysème pulmonaire post-tabagique qu'une perturbation de l'équilibre Nrf2-Keapl-Bachl, avec diminution de l'expression nucléaire de Nrf2 dans les macrophages alvéolaires, est associée à une diminution parallèle de l'expression de nombreuses enzymes antioxydantes, corrélée à la sévérité de la maladie et aux marqueurs de stress oxydant [16]. L'exposition à la fumée de tabac de macrophages in vitro induit une expression et une activité biphasiques (augmentation précoce et diminution tardive) des enzymes antioxydantes liées à une régulation spécifique de Nrf2/Keapl-Bachl via les voies de signalisation des MAP-kinases ERK et JNK [17]. Ces mécanismes pourraient expliquer les altérations observées au cours de l'emphysème pulmonaire post-tabagique chez l'homme. Un déséquilibre de la voie Nrf2/ Keapl-Bachl est probablement présent dans d'autres types cellulaires impliqués dans la physiopathologie de la BPCO. Ainsi, une diminution de la protéine DJ-1, qui stabilise Nrf2, a été mise en évidence dans le poumon 

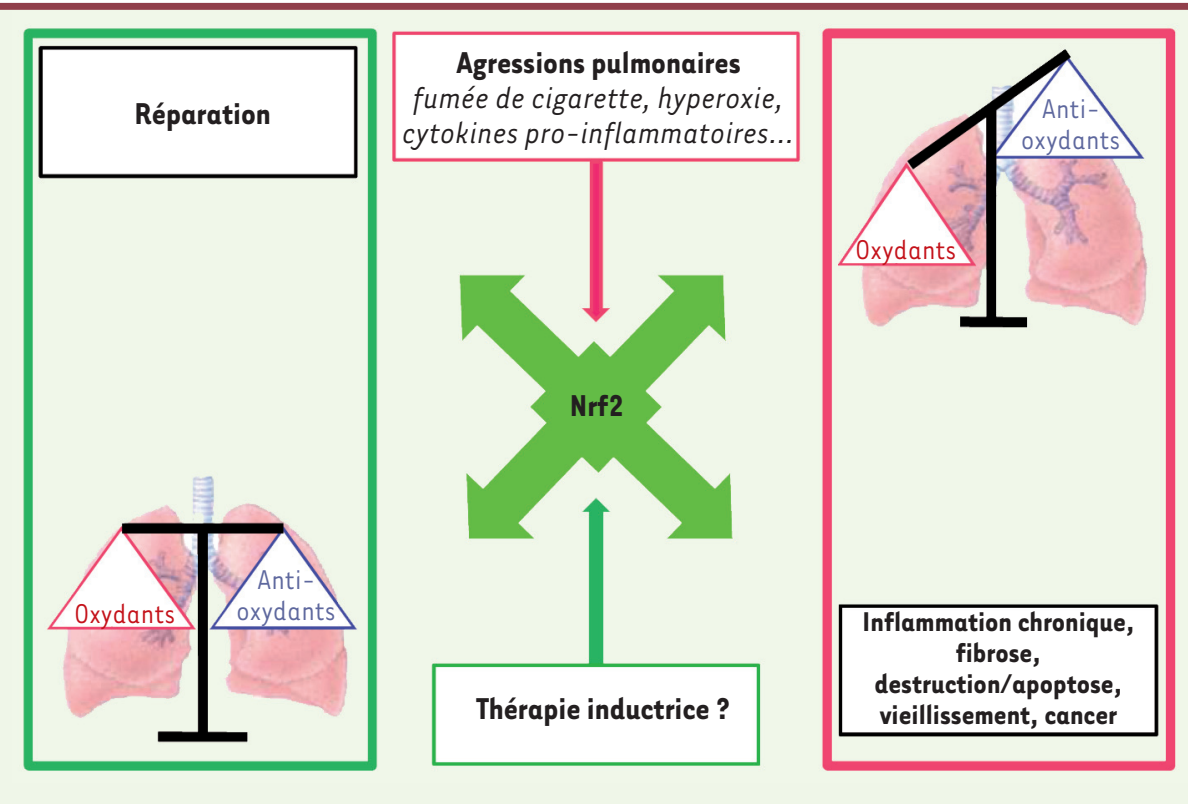

ment [25]. II faut noter que Nrf2 était absent des foyers fibroblastiques dans cette étude. Nos travaux suggèrent un rôle de Nrf2 dans la modulation du phénotype cellulaire au cours des processus fibrosants [26] et son intérêt probable en tant que cible thérapeutique. Dans les agressions alvéolaires aiguës, plusieurs modèles animaux ont montré le rôle du facteur Nrf2 [9] (Tableau II). Chez l'homme, un polymorphisme fonctionnel de Nrf2 a été associé à un risque accru de syndrome de détresse respiratoire aiguë [27].

\section{Asthme et autres pathologies respiratoires}

niveau pulmonaire au cours des agressions.

En dehors du modèle d'asthme chez la souris $\mathrm{Nrf2}^{-/-}$, peu d'étu-

des patients présentant une BPCO [18]. De même, Malhotra et al. ont montré que l'activité du protéasome qui dépend de Nrf2 est diminuée au cours de la BPCO et s'accompagne d'un stress du réticulum endoplasmique avec augmentation de l'apoptose qui pourrait participer à la progression de la maladie [19]. Récemment, un polymorphisme de Nrf2 et Keapl a été associé au niveau d'obstruction bronchique mesuré lors d'explorations fonctionnelles respiratoires dans la population générale [20].

\section{Agression alvéolaire}

La physiopathologie de la fibrose pulmonaire idiopathique (FPI), pathologie incurable, est mal connue. L'hypothèse principale est celle d'une lésion initiale de l'épithélium alvéolaire entraînant une augmentation des médiateurs qui stimulent l'inflammation et la prolifération des fibroblastes, associée à un échec des processus de réparation [21]. De nombreuses études ont montré l'importance du stress oxydant et sa participation à la physiopathologie de la fibrose pulmonaire [4]. On sait que les ROS sont des modulateurs de la différenciation cellulaire [2]. L'expression de la NOX4/NADPH oxydase à l'origine d'une augmentation des ROS est augmentée dans les fibroblastes pulmonaires de patients atteints de FPI [22]. Cette augmentation des ROS a été impliquée dans la différenciation du fibroblaste en myofibroblaste pulmonaire, celle-ci pouvant contribuer au développement de la fibrose [22]. De manière intéressante, Nrf2 est une cible d'action du KGF (keratinocyte growth factor), facteur de croissance pour les cellules épithéliales alvéolaires aux propriétés antifibrosantes [23]. À l'inverse, le TGF- $\beta$ (transforming growth factor), facteur profibrosant, supprime l'expression de Nrf2 [24]. L'augmentation de l'expression de Nrf2 et Keapl dans l'épithélium alvéolaire hyperplasique de sujets présentant une fibrose pulmonaire idiopathique a été rapportée récem- des cliniques sur la voie de signalisation Nrf2/KeaplBachl sont disponibles dans cette pathologie [28]. En revanche, il faut noter qu'un essai thérapeutique utilisant un inducteur de Nrf2 est en cours ${ }^{1}$.

Au cours de la mucoviscidose, il a été montré que le CDDO (acide 2-cyano-3,12-dioxooléana-1,9(11)-dien-28oïque), un triterpénoïde de synthèse inducteur de Nrf2, limite l'inflammation [29]. Cependant, l'inhibition de la transcription du gène CFTR (cystic fibrosis transmembrane conductance regulator) par Nrf2 dans une autre étude suggère des mécanismes plus complexes [30].

Au cours des cancers, le rôle de Nrf2 est actuellement incertain [31]. Différents arguments suggèrent que les gènes induits par Nrf2 ont un rôle protecteur vis-à-vis du cancer [32] et que le défaut de Nrf2 contribue à une susceptibilité accrue des patients atteints de BPCO post-tabagique au développement d'un cancer pulmonaire [33]. Ainsi, les gènes codant pour des antioxydants sont diminués dans l'épithélium pulmonaire de patients fumeurs atteints de cancer [34]. Une susceptibilité accrue de l'ADN aux mutations spontanées et induites par le benzopyrène dans le poumon de souris $\mathrm{Nrf2}^{-/-}$a été rapportée [35]. De même, la diminution de la protection contre le cancer observée chez des souris $\mathrm{Nrf2}^{-/-}$en restriction calorique suggère que la plupart des effets bénéfiques de la restriction calorique sur les processus de carcinogenèse empruntent la voie Nrf2 [36]. D'autre part, l'implication de Nrf2 dans la résistance des cellu-

${ }^{1}$ http://clinicaltrials.gov 


\begin{tabular}{|c|c|}
\hline & Mécanismes d'action \\
\hline Curcumin & $\begin{array}{l}\nearrow \text { p38, } \nearrow \text { translocation nucléaire de Nrf2 } \\
\nearrow \text { Activité de } \mathrm{H} 0-1 \text { dans les monocytes humains }\end{array}$ \\
\hline Resvératrol & $\begin{array}{l}\nearrow \text { Production du glutathion réduit } \\
\searrow \text { Libération de cytokines pro-inflammatoires } \\
\nearrow \text { Translocation nucléaire de Nrf2 } \\
\nearrow \text { Activité de } \mathrm{HO}-1\end{array}$ \\
\hline Quercétine & $\begin{array}{l}\nearrow \text { GSH intracellulaire } \\
\searrow \text { Production de ROS } \\
\nearrow \text { Translocation nucléaire de Nrf2 } \\
\nearrow \text { Activité de } \mathrm{HO}-1\end{array}$ \\
\hline $\begin{array}{l}\text { Catéchine (Epigallocatechin } \\
\text { gallate) }\end{array}$ & $\begin{array}{l}\nearrow \text { p38, }{ }_{\text {Akt }} \\
\nearrow \text { Translocation nucléaire de Nrf2 } \\
\nearrow \text { Activité de } \mathrm{HO} \text {-1 dans les lymphocytes B }\end{array}$ \\
\hline $\begin{array}{l}\text { Terpènoïdes } \\
\text { (lycopène, lutéïne, zéaxanthine, } \\
\beta \text {-carotène, CDDO, ginkgo biloba) }\end{array}$ & $\begin{array}{l}\searrow \text { Peroxydation lipidique } \\
\searrow \mathrm{O}_{2}^{-} \\
\nearrow \text { Phosphorylation de MAPK } \\
\nearrow \text { Translocation nucléaire de Nrf2 } \\
\nearrow \text { HO-1 }\end{array}$ \\
\hline Isothiocyanate (sulforaphane) & $\begin{array}{l}\searrow \text { p38, } \nearrow \text { translocation nucléaire de } \mathrm{Nrf2} \\
\nearrow \text { Activité de } \mathrm{HO}-1 \text { dans les hépatocytes humains }\end{array}$ \\
\hline
\end{tabular}

Tableau III. Activateurs potentiels de la voie Nrf2.

les cancéreuses aux chimiothérapies anticancéreuses est bien connue. Récemment une mutation de Keapl à l'origine d'une augmentation de l'expression de Nrf2 a été détectée dans des lignées de cancer du poumon, et est impliquée dans la résistance aux chimiothérapies [33]. De même, une mutation de Nrf2 s'accompagnant d'un défaut de son élimination par le protéasome a été identifiée chez $10 \%$ des patients atteints de cancer du poumon et est associée au processus de cancérisation [37]. De manière intéressante, la suppression transitoire de l'expression de Nrf2 dans les cellules cancéreuses pourrait diminuer leur chimiorésistance en affectant l'expression des gènes induits par Nrf2 [37]. Cette dualité du rôle de Nrf2 dans les cancers incite à la prudence dans l'utilisation de thérapies inductrices de ce facteur. En effet, le potentiel cancérigène de doses élevées d'antioxydants phénoliques activateurs de Nrf2 a été signalé $[7,38]$. L'activation de la voie Nrf2 dans les cellules cancéreuses pourrait engendrer des effets potentiellement délétères en particulier dans le cancer du poumon qui est souvent associé à la BPCO. En effet, l'activation de Nrf2 pourrait protéger les cellules cancéreuses des effets toxiques potentiels du stress oxydant et des chimiothérapies [31].

\section{La voie Nrf2 : future cible thérapeutique en pathologie respiratoire?}

Bien que largement testés dans les maladies respiratoires, les suppléments alimentaires et les molécules antioxydantes visant à augmenter les défenses antioxydantes non enzymatiques n'ont pas fait la preuve de leur intérêt thérapeutique. La plupart des études décrites précédemment suggèrent que le facteur de transcription Nrf2 pourrait constituer une cible intéressante pour de nouvelles stratégies thérapeutiques ou de prévention, en restaurant les antioxydants endogènes et les enzymes de détoxification (Tableau III) [5]. La première approche consiste en l'utilisation de composés comme les terpènoïdes, les flavonoïdes et les isothiocyanates qui activent la transcription des EPII via I'ARE en favorisant la dissociation du complexe Nrf2-Keapl et/ou en augmentant l'activité transcriptionelle du complexe formé par Nrf2 et les autres facteurs de transcription [5]. L'activation de la voie Nrf2 serait responsable des effets bénéfiques de composés alimentaires comme le curcumin du curry, l'épicatéchine du cacao et du thé, le sulforaphane des brocolis, les terpénoïdes et flavones extraits de Ginkgo biloba et les isoflavones du soja dans les maladies neurologiques $[39,40]$, cardiovasculaires [41] et pulmonaires comme la BPCO et l'emphysème [42, 43]. La deuxième approche est la surexpression de Nrf2, grâce à l'utilisation de virus utilisés comme vecteurs responsables de l'infection spécifique d'un tissu in vivo, ou à l'infection de cellules ex vivo greffées ensuite au patient. Selon les résultats des études in vitro et chez l'animal, le CDDO et ses dérivés triterpénoïdes de synthèse promettent des applications potentielles dans le traitement des maladies rénales, neurodégénératives [44], cardiaques [45] et pulmonaires [46]. Cette molécule a d'ailleurs fait l'objet d'essais cliniques de phase I dans le traitement des leucémies et tumeurs solides et est actuellement utilisée dans des essais de phase IIb dans le traitement de l'insuffisance rénale chronique dans le diabète de type $2^{2}$.

\section{Conclusion}

Le stress oxydant et la voie de signalisation Nrf2/KeaplBachl sont impliqués dans la physiopathologie de nombreuses pathologies respiratoires. Même si plusieurs

${ }^{2}$ http://clinicaltrials.gov. 
essais cliniques sont en cours, l'intérêt d'un traitement activant la voie Nrf2 et ciblant les patients chez lesquels on observe un déficit en Nrf2 reste à démontrer (Figure 2). Il est probable qu'un tel traitement ne pourrait être qu'adjuvant dans certaines pathologies respiratoires compte tenu de l'absence de spécificité du stress oxydant. D'autres études sont nécessaires pour préciser le rôle de Nrf2 en fonction du stade de la maladie, des facteurs de risque associés ou des comorbidités. $\diamond$

\section{SUMMARY}

\section{Protective role of Nrf2 in the lungs against oxidative airway diseases}

Airways are continually exposed to multiple inhaled oxidants and protect themselves with cellular and extracellular antioxidants throughout the epithelial lining fluid and tissues. Oxidative stress, resulting from the increased oxidative burden and decreased level of antioxidant proteins, is involved in cellular and tissue damage related to the pathogenesis of many acute and chronic respiratory diseases. Evidence suggested that nuclear factor erythroid-2-related factor 2 (Nrf2), a transcription factor that controls antioxidant response element (ARE)regulated antioxidant and cytoprotective genes has an essential protective role in the lungs against oxidative airway diseases. Therefore, Nrf2 promises to be an attractive therapeutic target for intervention and prevention strategies in respiratory diseases. We have reviewed major findings on the mechanisms of lung protection against oxidative stress by Nrf2 and the current literature suggesting that Nrf2 is a valuable therapeutic target. $\diamond$

\section{CONFLIT D'INTÉRÊT}

Les auteurs déclarent n'avoir aucun conflit d'intérêts concernant les données publiées dans cet article.

\section{REMERCIEMENTS}

Marcel Bonay a obtenu un contrat de recherche du fonds de dotation Recherche en santé respiratoire et une subvention Legs Poix de la chancellerie des Universités de Paris.

\section{RÉFÉRENCES}

1. Bonay M, Aubier M. Pollution atmosphérique et maladies respiratoires allergiques. Med Sci (Paris) $2007 ; 23: 187-92$.

2. Yamamoto T, Sakaguchi N, Hachiya M, et al. Role of catalase in monocytic differentiation of U937 cells by TPA: hydrogen peroxide as a second messenger. Leukemia $2009 ; 23$ : 761-9.

3. Rangasamy T, Williams MA, Bauer $S$, et al. Nuclear erythroid 2 p45-related factor 2 inhibits the maturation of murine dendritic cells by ragweed extract. Am J Respir Cell Mol Biol $2010 ; 43: 276-85$.

4. Walters DM, Cho HY, Kleeberger SR. Oxidative stress and antioxidants in the pathogenesis of pulmonary fibrosis: a potential role for Nrf2. Antioxid Redox Signal 2008 ; 10 : 321-32.

5. Boutten A, Goven D, Boczkowski J, Bonay M. Oxidative stress targets in pulmonary emphysema: focus on the Nrf2 pathway. Expert Opin Ther Targets $2010 ; 14: 329-46$.

6. Valko M, Leibfritz D, Moncol J, et al. Free radicals and antioxidants in normal physiological functions and human disease. Int J Biochem Cell Biol 2007 ; 39 : 44-84.

7. Leonarduzzi G, Sottero B, Poli G. Targeting tissue oxidative damage by means of cell signaling modulators: the antioxidant concept revisited. Pharmacol Ther $2010 ; 128: 336-74$.

8. Sykiotis GP, Bohmann D. Stress-activated cap'n'collar transcription factors in aging and human disease. Sci Signal 2010 ; 3 : re3.

9. Kensler TW, Wakabayashi N, Biswal S. Cell survival responses to environmental stresses via the Keap1-Nrf2-ARE pathway. Annu Rev Pharmacol Toxicol 2007 ; 47 : 89-116.

10. Gong X, Kole L, Iskander K, Jaiswal AK. NRH: quinone oxidoreductase 2 and NAD(P)H: quinone oxidoreductase 1 protect tumor suppressor $\mathrm{p} 53$ against 20 s proteasomal degradation leading to stabilization and activation of p53. Cancer Res $2007 ; 67: 5380-8$.
11. Morse D, Choi AM. Heme oxygenase-1: from bench to bedside. Am J Respir Crit Care Med $2005 ; 172: 660-70$.

12. Malhotra D, Portales-Casamar $\varepsilon$, Singh A, et al. Global mapping of binding sites for Nrf2 identifies novel targets in cell survival response through ChIPSeq profiling and network analysis. Nucleic Acids Res 2010 ; 38 : 5718-34.

13. Yoshida T, Tuder RM. Pathobiology of cigarette smoke-induced chronic obstructive pulmonary disease. Physiol Rev 2007 ; 87 : 1047-82.

14. Shaykhiev R, Krause A, Salit J, et al. Smoking-dependent reprogramming of alveolar macrophage polarization: implication for pathogenesis of chronic obstructive pulmonary disease. J Immunol 2009 ; 183 : 2867-83.

15. Hogg JC, Timens W. The pathology of chronic obstructive pulmonary disease. Annu Rev Pathol 2009 ; 4 : 435-59.

16. Goven D, Boutten A, Lecon-Malas V, et al. Altered Nrf2/Keapl-Bachl equilibrium in pulmonary emphysema. Thorax $2008 ; 63: 916-924$.

17. Goven D, Boutten A, Lecon-Malas V, et al. Prolonged cigarette smoke exposure decreases heme oxygenase- 1 and alters Nrf2 and Bachl expression in human macrophages: roles of the MAP kinases ERK(1/2) and JNK. FEBS Lett 2009 ; 583 : 3508-18.

18. Malhotra D, Thimmulappa R, Navas-Acien A, et al. Decline in NRF2regulated antioxidants in chronic obstructive pulmonary disease lungs due to loss of its positive regulator, DJ-1. Am J Respir Crit Care Med 2008; 178 : 592-604.

19. Malhotra D, Thimmulappa R, Vij N, et al. Heightened endoplasmic reticulum stress in the lungs of patients with chronic obstructive pulmonary disease: the role of Nrf2-regulated proteasomal activity. Am J Respir Crit Care Med $2009 ; 180: 1196-207$.

20. Siedlinski M, Postma DS, Boer JM, et al. Level and course of FEVl in relation to polymorphisms in NFE2L2 and KEAP1 in the general population. Respir Res $2009 ; 10: 73$.

21. Strieter RM, Mehrad B. New mechanisms of pulmonary fibrosis. Chest 2009 ; $136: 1364-70$.

22. Hecker $L$, Vittal R, Jones $T$, et al. NADPH oxidase- 4 mediates myofibroblast activation and fibrogenic responses to lung injury. Nat Med $2009 ; 15: 1077-81$.

23. Braun S, Hanselmann C, Gassmann MG, et al. Nrf2 transcription factor, a novel target of keratinocyte growth factor action which regulates gene expression and inflammation in the healing skin wound. Mol Cell Biol 2002 ; $22: 5492-505$.

24. Bakin AV, Stourman NV, Sekhar KR, et al. Smad3-ATF3 signaling mediates TGF-beta suppression of genes encoding Phase II detoxifying proteins. Free Radic Biol Med $2005 ; 38: 375-87$.

25. Mazur W, Lindholm P, Vuorinen K, et al. Cell-specific elevation of NRF2 and sulfiredoxin- 1 as markers of oxidative stress in the lungs of idiopathic pulmonary fibrosis and non-specific interstitial pneumonia. Apmis 2010 ; $118: 703-12$.

26. Artaud-Macari $\varepsilon$, Goven D, Brayer $S$, et al. Modulation of fibroblast phenotype in idiopathic pulmonary fibrosis: role of Nrf2. Am J Respir Crit Care Med 2011 ; 183 : A5989.

27. Marzec JM, Christie JD, Reddy SP, et al. Functional polymorphisms in the transcription factor NRF2 in humans increase the risk of acute lung injury. Faseb J $2007 ; 21: 2237-46$.

28. Kim SH, Choi GS, Ye YM, et al. Toluene diisocyanate (TDI) regulates haem oxygenase-1/ferritin expression: implications for toluene diisocyanateinduced asthma. Clin Exp Immunol 2010 ; $160: 489-97$.

29. Nichols DP, Ziady AG, Shank SL, et al. The triterpenoid CDDO limits inflammation in preclinical models of cystic fibrosis lung disease. Am J Physiol Lung Cell Mol Physiol $2009 ; 297$ : L828-36.

30. Rene C, Lopez $\varepsilon$, Claustres M, et al. NF- $\varepsilon 2$-related factor 2, a key inducer of antioxidant defenses, negatively regulates the CFTR transcription. Cell Mol Life Sci $2010 ; 67: 2297-309$.

31. Ohta T, lijima K, Miyamoto M, et al. Loss of Keapl function activates Nrf2 and provides advantages for lung cancer cell growth. Cancer Res 2008 ; 68 : 1303-9.

32. Cho HY, Reddy SP, Kleeberger SR. Nrf2 defends the lung from oxidative stress. Antioxid Redox Signal $2006 ; 8: 76-87$.

33. Boutten A, Goven D, Artaud-Macari $\varepsilon$, et al. NRF2 targeting: a promising therapeutic strategy in chronic obstructive pulmonary disease. Trends Mol Med $2011 ; 17: 363-71$.

34. Spira A, Beane JE, Shah V, et al. Airway epithelial gene expression in the diagnostic evaluation of smokers with suspect lung cancer. Nat Med 2007 ; $13: 361-6$.

35. Aoki Y, Hashimoto AH, Amanuma K, et al. Enhanced spontaneous and benzo(a)pyrene-induced mutations in the lung of Nrf2-deficient gpt delta mice. Cancer Res 2007 ; 67 : 5643-8. 


\section{RÉFÉRENCES}

36. Martin-Montalvo A, Villalba JM, Navas P, de Cabo R. NRF2, cancer and calorie restriction. Oncogene $2010 ; 30: 505-20$.

37. Shibata T, Ohta T, Tong KI, et al. Cancer related mutations in NRF2 impair its recognition by Keapl-Cul3 E3 ligase and promote malignancy. Proc Natl Acad Sci USA 2008 ; 105 : 13568-73.

38. Hayes JD, MCMahon M. NRF2 and KEAP1 mutations: permanent activation of an adaptive response in cancer. Trends Biochem Sci $2009: 34: 176-88$.

39. Niso-Santano M, Gonzalez-Polo RA, Bravo-San Pedro JM, et al. Activation of apoptosis signalregulating kinase 1 is a key factor in paraquat-induced cell death: modulation by the Nrf2/Trx axis. Free Radic Biol Med 2010 ; 48 : 1370-81.

40. Shah ZA, Li RC, Ahmad AS, et al. The flavanol (-)-epicatechin prevents stroke damage through the Nrf2/HOl pathway. J Cereb Blood Flow Metab 2010 ; 30 : 1951-61.

41. Mann GE, Bonacasa B, Ishii T, Siow RC. Targeting the redox sensitive Nrf2-Keapl defense pathway in cardiovascular disease: protection afforded by dietary isoflavones. Curr Opin Pharmacol 2009 ; $9: 139-45$.

42. Yoon HY, Kang NI, Lee HK, et al. Sulforaphane protects kidneys against ischemia-reperfusion injury through induction of the Nrf2-dependent phase 2 enzyme. Biochem Pharmacol 2008 ; 75 : 2214-23.
43. Hsu CL, Wu YL, Tang GJ, et al. Ginkgo biloba extract confers protection from cigarette smoke extract-induced apoptosis in human lung endothelial cells: role of heme oxygenase-1. Pulm Pharmacol Ther $2009 ; 22: 286-96$.

44. Yang L, Calingasan NY, Thomas B, et al. Neuroprotective effects of the triterpenoid, CDDO methyl amide, a potent inducer of Nrf2-mediated transcription. PLoS One 2009; 4 : e5757.

45. Ichikawa T, Li J, Meyer CJ, et al. Dihydro-CDD0-trifluoroethyl amide (dh404), a novel Nrf2 activator, suppresses oxidative stress in cardiomyocytes. PLoS One $2009 ; 4$ : e8391.

46. Sussan TE, Rangasamy T, Blake DJ, et al. Targeting Nrf2 with the triterpenoid CDDO-imidazolide attenuates cigarette smoke-induced emphysema and cardiac dysfunction in mice. Proc Natl Acad Sci USA $2009 ; 106: 250-5$.

\section{Collection SCIENCE ET BIOMÉDECINE}

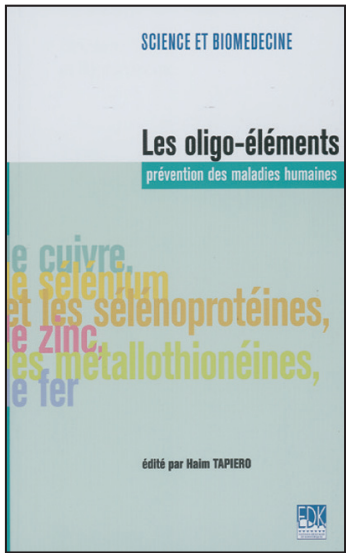

ISBN : 2-84254-107-3 64 pages

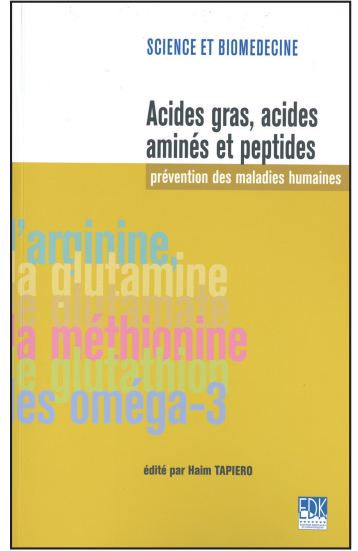

ISBN : 2-84254-108-1 80 pages

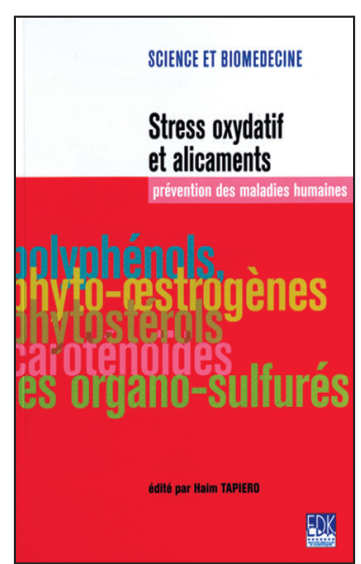

ISBN : 2-84254-111-1 86 pages

\section{Bon de commande}

À retourner à EDK, 25, rue Daviel - 75013 Paris

Tél. : 0158101905 - Fax : 0143293262 - E-mail : edk@edk.fr

NOM : Prénom :

Adresse :

Code postal :

Ville :

Pays :

Fonction :

Je souhaite recevoir l'ouvrage Les oligo-éléments : $10 €+3 €$ de port $=\mathbf{1 3} €$ TTC

Je souhaite recevoir l'ouvrage Acides gras, acides aminés et peptides : $12 €+3 €$ de port $=15 €$ TTC

Je souhaite recevoir l'ouvrage Stress oxydatif et alicaments : $14 €+3 €$ de port $=17 €$ TTC

$\square$ exemplaire, soit un total de $€$

\section{Carte $n^{\circ}$}

chèque, à l'ordre de $\mathbf{E} \mathbf{D} \mathbf{K}$

Par carte bancaire :

$\square$ Visa $\square$ Eurocard/Mastercard

Date d'expiration :

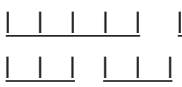

Signature :

$\mathrm{N}^{\circ}$ de contrôle au dos de la carte :

TIRÉS À PART

M. Bonay 